\title{
ARROW-Type Vertical Coupler Filter: Design and Fabrication
}

\author{
S. T. Chu, Member, IEEE, W. Pan, S. Sato, Brent E. Little, Member, IEEE, T. Kaneko, \\ and Y. Kokubun, Member, IEEE
}

\begin{abstract}
Vertical coupler filters (VCF) exhibiting narrow bandwidth and low sidelobe levels have been designed and demonstrated. Narrow bandwidth filter response is achieved due to the strong asymmetry between the waveguides of the filter and the nondispersive characteristics of the anitresonant reflecting optical waveguide (ARROW) structure. An ARROW-type VCF with a conventional parallel coupled directional coupler configuration with a full width at half-maximum (FWHM) of $1.36 \mathrm{~nm}$ and a maximum sidelobe level of $-8.5 \mathrm{~dB}$ was fabricated using a coumpound glass consisting of $\mathrm{SiO}_{2}$ and $\mathrm{SiO}_{2} / \mathrm{Ta}_{2} \mathrm{O}_{5}$. The filter sidelobe levels were then further suppressed by using an $\mathrm{X}$-crossing arrangement to provide coupling strength apodization along the device. The sidelobe levels of this modified $X$-crossing filter were suppressed to below $-23 \mathrm{~dB}$ and the measured FWHM was $3.9 \mathrm{~nm}$. The central wavelength of the reported filters are in the $1.55 \mu \mathrm{m}$ region. The measured results are in good agreement with theoretical results from an analysis procedure that combines the coupled mode theory with the finite difference complex mode solver.
\end{abstract}

Index Terms-Add-drop filters, optical filter, sidelobe suppression, wavelength division multiplexed (WDM) components.

\section{INTRODUCTION}

$\mathbf{O}$ PTICAL wavelength add-drop filters, which provide channel selective functions, are essential elements in wavelength division multiplexed (WDM) access networks. The characteristics of these filters limit the capacity of the network, in terms of channel spacing and channel density. One particularly attractive add-drop filter configuration is the vertically coupled filter (VCF) [1]. In contrast to the planar version, where the two waveguides are placed side-by-side, the waveguides of the VCF are placed one on top of the other. In this configuration one is less restricted in the choice of geometry or material composition in comparison to the planar version. Thus, it becomes easier to have very dissimilar waveguides which is the key to achieving very narrow bandwidth characteristics. The narrowing of the bandwidth can further be enhanced with grating assisted coupling or when

Manuscript received August 24, 1998; revised January 18, 1999.

S. T. Chu, W. Pan, and S. Sato are with the Kanagawa Academy of Science and Technology, 3D Microphotonics Project, Kawasaki 213-0012 Japan.

B. E. Little is with the Research Laboratory of Electronics, and the Department of Electrical Engineering and Computer Sciences, Massachusetts Institute of Technology, Cambridge MA 02139 USA. He is also with the Laboratory for Physical Sciences, University of Maryland at College Park, College Park, MD 20742 USA

T. Kaneko and Y. Kokubun are with the Department of Electrical and Computer Engineering, Yokohama National University, Yokohama 240-8501 Japan.

Publisher Item Identifier S 0733-8724(99)02683-3. the nondispersive ARROW waveguide is used as one of the coupled waveguides [2].

A number of high-performance VCF's have been fabricated [3], [4] with semiconductor materials. Although these VCF's are very narrowband and can operate over a relatively large tunning range, the waveguides are not fiber matched and spot size transformers are needed for their deployment in the WDM systems. Futhermore, these devices were optimized only for their bandwidth performance and the sidelobe levels were shown to be relatively high, typically higher than $-10 \mathrm{~dB}$. Since these filters consist of waveguides in which the upper waveguide runs completely parallel to the lower waveguide, one is not free to introduce coupling strength apodization, which would otherwise enhance the sidelobe suppression.

It is well known that the sidelobe level of the filter response depends on the coupling strength apodization along the length of the device. In order to obtain high sidelobe suppression, the coupling strength of the filter must vary smoothly along the waveguide, decreasing toward either end. Any abrupt change in the coupling distribution raises the sidelobe level.

The coupling interaction can be controlled by adjusting waveguide separation or by applying a weighting function on the applied electrodes of the electrooptic devices. The use of weighted coupling strength distribution to suppress the sidelobe level of the filter response has been demonstrated recently by refs. Smith and Johnson [5], Croston et al. [6], and Little et al. [7] has outlined the tradeoffs between filter bandwidth and sidelobe level and derived the limiting performance in terms of sidelobe suppression of the coupler filters and compared the sidelobe levels of the various coupling strength apodization shapes.

Recently, we reported a VCF configuration where the two waveguides are crossed in a $X$ type shape, which achieves a sidelobe suppression level better than $-17 \mathrm{~dB}$ [8]. The ARROW waveguide in our earlier reported filter was fabricated using a wet-etching technique, which introduces a small slope at the side of the core/cladding interface. The VCF fabricated by wet etching presents three drawbacks. First the width of the waveguides cannot be precisely controlled. Second, the edge of the SLC is not vertical and the cross section of the upper waveguide is not rectangular, which also introduces difficulties in the exact analysis. Last, the wet-etching process leaves a trench on the top surface of the ARROW waveguide along the lateral core/cladding boundaries. The trench distorts the shape of the upper waveguide in the region where it crosses over the core/cladding boundary of the lower waveguide 
with the amount of distortion depends on the crossing angle. In this paper, modified ARROW-type VCF's are fabricated by an improved planarization technique, which maximizes the flatness on the top of the ARROW waveguide, and thus minimizes the cross-over distortion. Using the improved fabrication procedure, the overall performance of the VCF has been improved.

In the theoretical design of the ARROW-type VCF, we developed an accurate and efficient approach that combines the coupled mode theory with a finite-difference mode solver. The finite-difference mode solver is used to calculate the complex propagation constant of the leaky modes in the ARROW waveguide. Using this analysis approach, the response of the VCF structure can be calculated very efficiently, provided that only the fundamental mode is launched. It is shown that this analysis method can accurately predict the performance of the device fabricated. In the fabrication step of the ARROWtype VCF, which consists of an ARROW and a conventional ridge or channel waveguide, a lift off process is developed for the fabrication of the SLC-ARROW structure. This reduces the trench at the core/cladding interfaces on the top surface of the ARROW waveguide to less than $0.1 \mu \mathrm{m}$. First a parallel ARROW-VCF is fabricated, which gives the narrowest bandwidth for a given device size [9]. Following this, the $\mathrm{VCF}$ employing an $\mathrm{X}$-crossing configuration is fabricated to investigate the role of apodization on sidelobe supression performance. In the crossing configuration, the coupling strength distribution resembles a hyperbolic secant function which provides much lower sidelobe levels than the conventional uniform coupling type VCF.

\section{ARROW-TyPE VCF WITH COUPLING STRENGTH APODIZATION}

One method to apply smooth apodization of the coupling strength in the VCF is to separate the two waveguides gradually. In this approach it is essential that the top surface of the lower waveguide layer remain flat and smooth. A structure which can satisfy this requirement is shown in Fig. 1. In the proposed filter, the lower waveguide is an SLC-ARROW waveguide where the lateral optical confinement is provided by the thin lower index layers placed at the center of the ARROW core layer. Since these layers can be made very thin (typically $0.5 \mu \mathrm{m}$ ), a relatively flat top surface which is suitable for the placement of the upper waveguide can be obtained. In addition, to provide a flat surface for the application of smooth apodization, the ARROW-type VCF offers several attractive features: The ARROW waveguide is well matched to a fiber mode and thus coupling loss can be kept small [10]. The ARROW mode is based on high reflection confinement, rather than total internal reflection, the dispersion of the mode can be made relatively flat [10]. Thus, when evanescently coupled to a highly dispersive channel waveguide, narrow band filter characteristics can be expected.

\section{Design AND ANALYSIS OF THE ARROW-Type VCF}

In the design of the filter, the coupled mode theory (CMT) was used in conjunction with a finite difference mode solver.

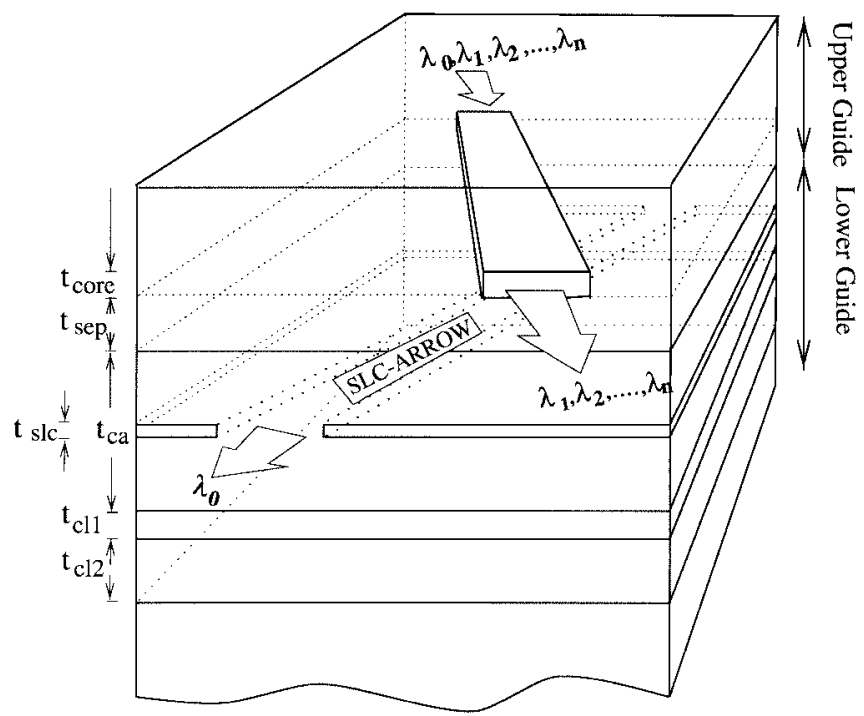

Fig. 1. The ARROW-type vertical coupler filter. Upper waveguide with any configuration can be placed on top of the flat SLC-ARROW structure.
SLC - ARROW

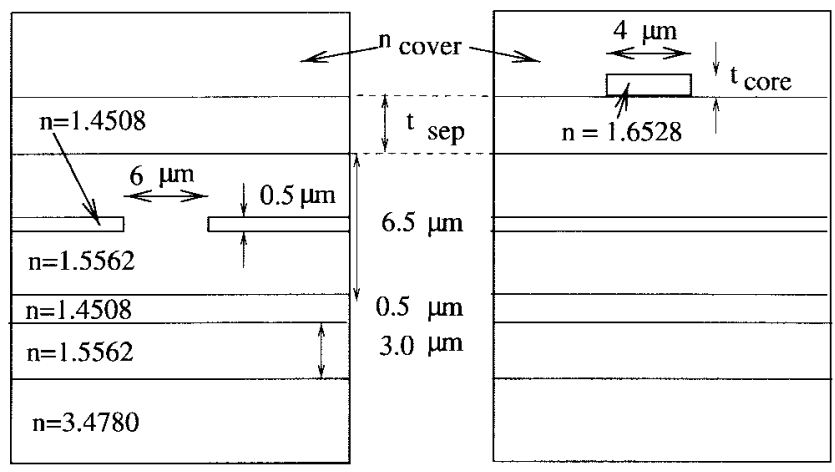

(a) (b)
Fig. 2. Reference waveguides used in the CMT analysis. (a) The ARROW with a thin lower index stripe for optical confinement in the lateral direction. (b) The upper waveguide consists of a buried channel or ridge waveguide sitting on top of a multilayered structure. The reference mode is the composite mode of the rectangular waveguide and the multilayered structure.

The mode solver is used to evaluate the exact propagation constants of the individual waveguides, in addition to their field profiles. The coupled mode theory is subsequently used to evaluate the coupling strength and wavelength response. In this manner, once the exact modes are solved, and the field profiles stored, the responses of filters with arbitrary apodization shapes can be evaluated and optimized quickly.

In the proposed VCF, a stripe lateral confinement (SLC) ARROW and a channel waveguide placed on top of a multilayered structure, as shown in Fig. 2, are used as the reference waveguides. Because of the leaky nature of the modes, the semivectorial version of the finite-difference mode solver [11] which can effectively calculate the complex propagation constants of these leaky modes were used to determine the propagation constants and field distributions of the reference modes.

The main consideration in the design of the ARROWtype VCF was to ensure that the VCF had to operate in the 


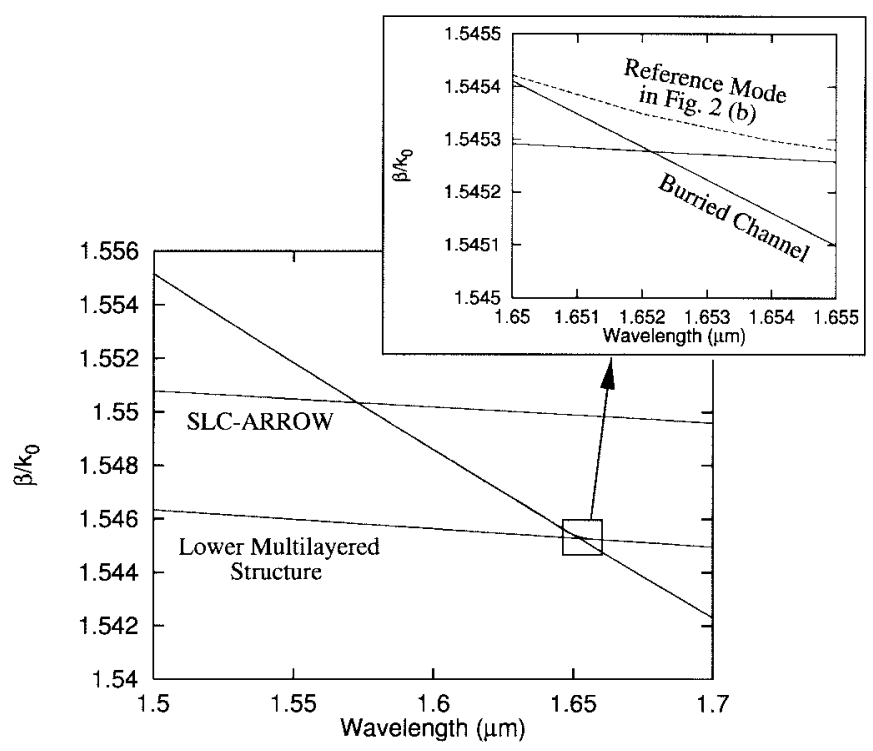

Fig. 3. Dispersion curves of the reference modes of the filter used in the CMT analysis with $t_{\text {core }}=0.70 \mu \mathrm{m}, t_{\text {sep }}=2.25 \mu \mathrm{m}$, and $n_{\text {cover }}=1.4508$. Due to the multilayer in Fig. 2 (b), the reference mode has a cutoff value the same as the lower multilayered structure.

singlemode region. However, higher order modes do exsit in the ARROW and these leaky modes are rejected only by loss discrimination. The dispersion of the waveguide in Fig. 2(b) differs from the conventional channel waveguide (without the lower multilayered structure) because its cutoff propagation constant depends on the lower multilayered structure. When the propagation constant of the upper waveguide is smaller than the propagation constant of the leaky modes in the lower structure, the power carried in the upper waveguide leaks into ARROW cladding and couples into the lower waveguide even when the cores of the upper and lower waveguides are separated far apart. Therefore, it is necessary to keep the cutoff wavelength outside of the wavelength range of operation in the design. Furthermore, the operation wavelength range depends on the dispersion of the channel, when the dispersion is increased (with large refractive index difference between the ARROW core and the channel waveguide core), the passband will be closer to the cutoff wavelength. Fig. 3 shows the typical dispersion characteristics of the transverse electric (TE) reference modes with its cutoff value, the waveguide parameters are the same as in Fig. 2, with $t_{\text {core }}=0.70 \mu \mathrm{m}$, $t_{\text {sep }}=2.25 \mu \mathrm{m}$ and $n_{\text {cover }}=1.4508$.

The dispersion of the upper waveguide can be increased by using a higher index core and/or using a ridge structure, where the $\mathrm{SiO}_{2}$ cover is removed or replaced by a lower index material. Fig. 4 shows a comparison of the dispersion curves between a buried channel and a ridge waveguide, both sitting on top of the same multilayered structure as in Fig. 2(b). In order to provide phase matching within the range of interest, the thickness of the ridge waveguide $t_{\text {core }}$ has to be increased to $0.86 \mu \mathrm{m}$.

Of all the waveguide dimensions, the size of the upper waveguide controls the passband location as compares to the size of the ARROW waveguide. For the structure in Fig. 2, a slight change of $t_{\text {core }}$ by $0.01 \mu \mathrm{m}$ results in a shift of

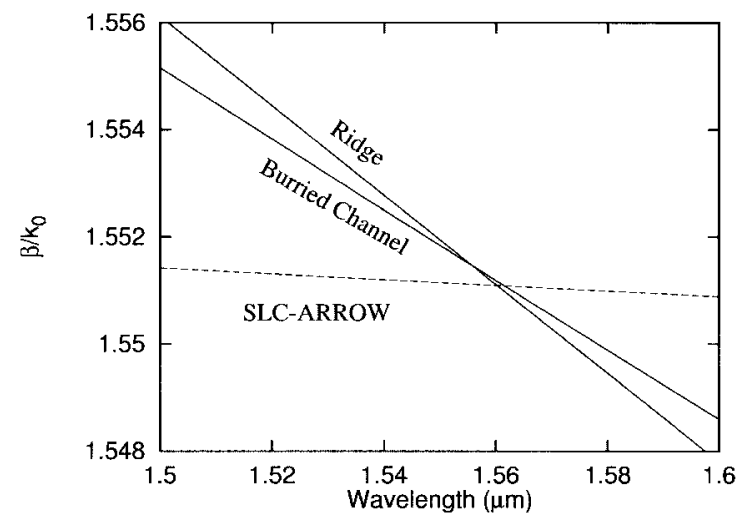

Fig. 4. Comparison between the dispersion of a buried channel and a ridge waveguide. The waveguide parameters of the waveguides are the same as in Fig. 3 but with $t_{\text {core }}=0.86 \mu \mathrm{m}$ and $n_{\text {cover }}=1.0$ for the ridge waveguide.

the passband location by $20 \mathrm{~nm}$, whereas a change of $t_{c a}$ by $0.10 \mu \mathrm{m}$ only shifts the passband by $1.5 \mathrm{~nm}$. Although film thickness can be controlled quite accurately by sputtering deposition, nonuniformity in the sputtered film can shift the location of the passband away from the desired location. Post processing such as trimming or tuning are necessary for the implementation of these types of devices. Recently, we have succeeded in the trimming of the filter passband location by 59 $\mathrm{nm}$ using an ultraviolet (UV) sensitive polymer as the cover layer of the ARROW VCF [12].

To obtain the overall filter response, the VCF are first subdivided into a cascade of $N$ independent sections with constant coupling coefficient $\kappa$, the overall transfer matrix at a single wavelength relating the power at the input port $A(0)$ to the final output port $A(L)$ is then solved from the cascaded transfer matrix equations.

In the calculation, the $\kappa$ 's of the transfer matrices are determined by numerical integration at a range of sampling separations using the calculated reference modes. The value of $\kappa$ at a particular separation can then be determined using interpolation. Once $\kappa(z)$ is evaluated, the CMT procedure can be used to determine the filter response of any apodization shape very efficiently.

The layout of the VCF's investigated in this paper is shown in Fig. 5. The lower ARROW of the VCF is kept straight along the length of the device, while the upper waveguide has a straight section of $L=1 \mathrm{~cm}$ with two curved sections with $R=25 \mathrm{~cm}$ at the ends for the detachment of the waveguides. The buffer thickness $t_{\text {sep }}$ and upper core thickness $t_{\text {core }}$ are adjusted for phase matching and maximum coupling efficiency for a given crossing angle $\theta$.

Fig. 6 shows the filter responses of the filters with $\theta=$ $0^{\circ}, 0.05^{\circ}$, and $0.10^{\circ}$. The waveguide parameters are the same as in Fig. 2, with $t_{\text {sep }}=2.25 \mu \mathrm{m}, t_{\text {core }}=0.85 \mu \mathrm{m}$, and $n_{\text {cover }}=1.0$. It can be seen that the sidelobe level is reduced when the waveguides are crossed, with the effect of the suppression increases with the crossing angle. For the stuctures used in this comparison, the sidelobe levels of the main sidelobe are $-8.5,-20,-40 \mathrm{~dB}$ at $\theta=0^{\circ}, 0.05^{\circ}$, and $0.10^{\circ}$, respectively. Because of the different filter shapes and the length of the effective coupling region, the bandwidth 


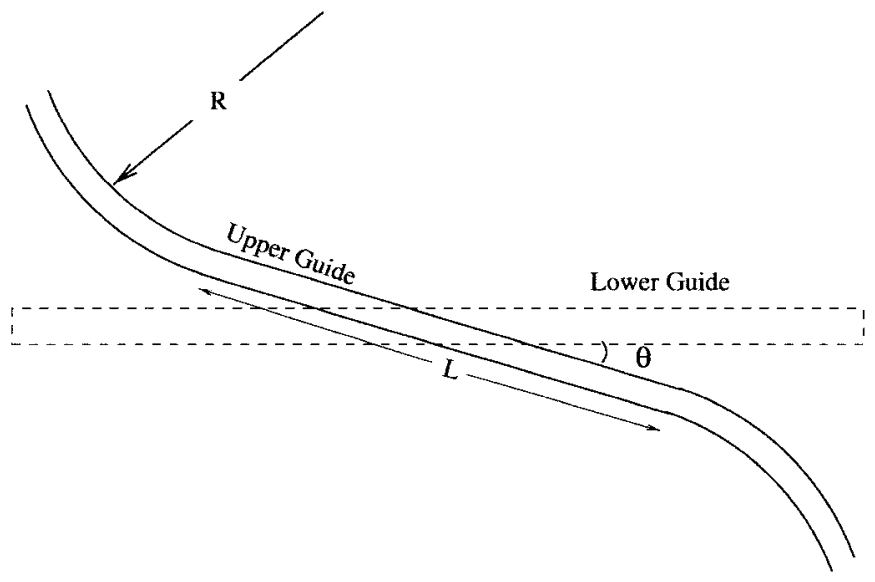

Fig. 5. Top views of the orientation of the VCF's investigated in this paper. The straight section $L$ is kept at $1 \mathrm{~cm}$ and $R=25 \mathrm{~cm}$ with $\theta$ adjusted to investigate its filter preformance.

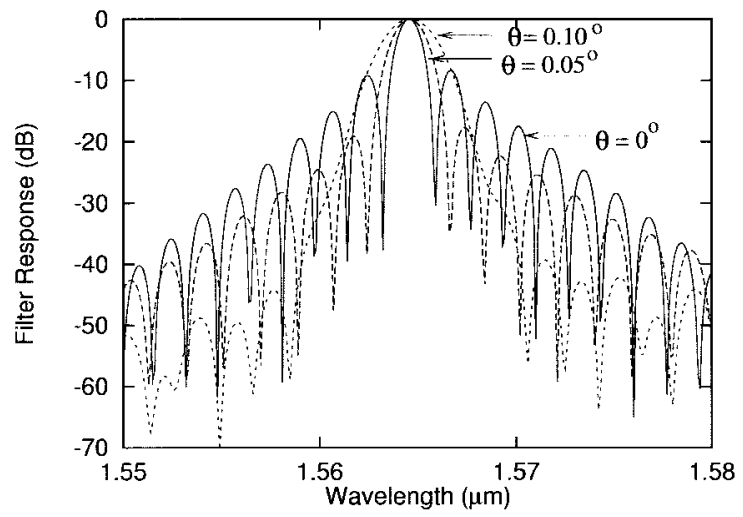

Fig. 6. Sidelobe level comparison of the filter configuration in Fig. 5 at vaious $\theta$. The waveguide parameters are the same as in Fig. 2 with $n_{\text {cover }}=1.0, t_{\text {core }}=0.86 \mu \mathrm{m}$ and $t_{\text {sep }}=2.25 \mu \mathrm{m}$.

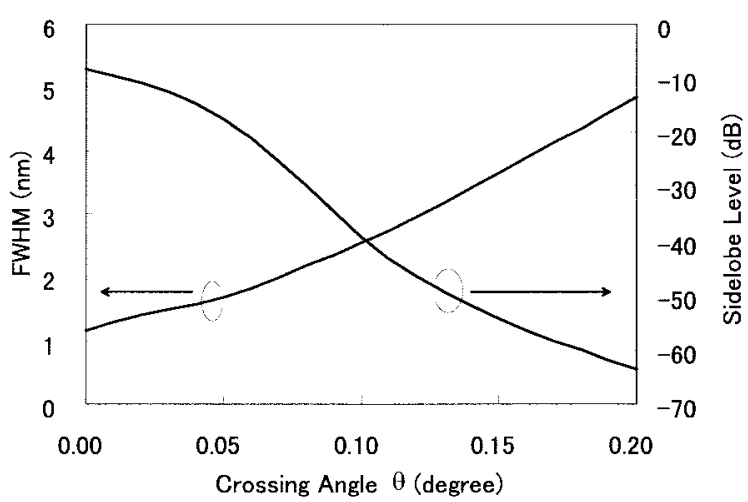

Fig. 7. Variation of the FWHM and sidelobe level with the crossing angle $\theta$. For a device at a fixed length, FWHM is compromized for the crossing angle.

of the filter increases with $\theta$. The relationship between the bandwidth and the crossing angle is shown in Fig. 7.

\section{DEVICE FABRicATION}

The waveguides of the ARROW-type VCF were deposited using $\mathrm{RF}$ sputtering of $\mathrm{SiO}_{2}$ and $\mathrm{Ta}_{2} \mathrm{O}_{5} / \mathrm{SiO}_{2}$ compound glass. The $\mathrm{Ta}_{2} \mathrm{O}_{5} / \mathrm{SiO}_{2}$ compound glass is used because its refractive index can be adjusted by the ratio of the composition of $\mathrm{Ta}_{2} \mathrm{O}_{5}$
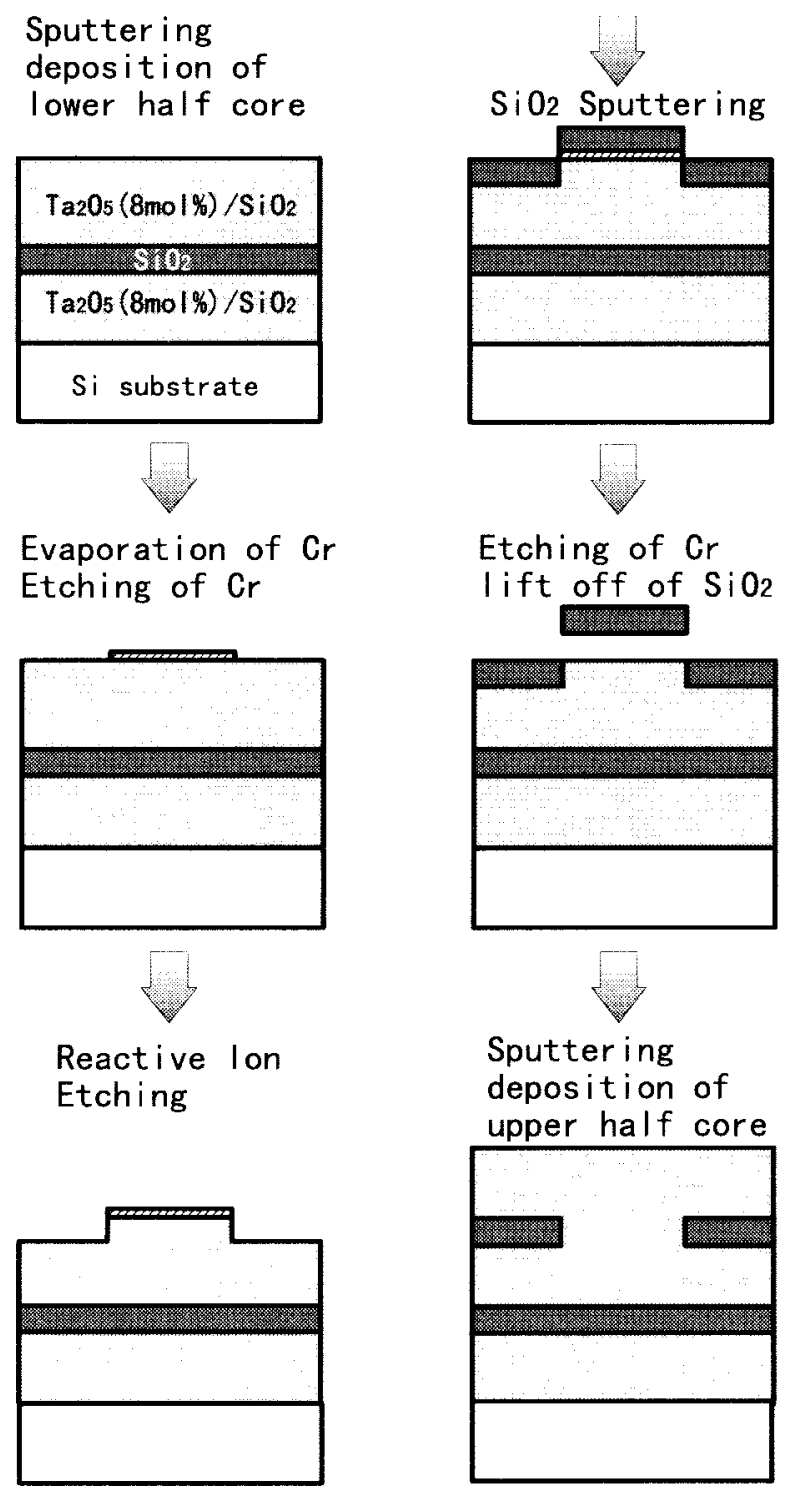

Fig. 8. Fabrication procedure of the lower SLC-ARROW structure. A lift-off process is required for the maximum flatness on top of its surface after fabrication.

and $\mathrm{SiO}_{2}$ and it can be dry etched with $\mathrm{CF}_{4}$. The fact that $\mathrm{Ta}_{2} \mathrm{O}_{5} / \mathrm{SiO}_{2}$ can be dry etched is essential for the planarization of the ARROW waveguide and the improvement of the VCF performance. In the fabricated devices, $\mathrm{Ta}_{2} \mathrm{O}_{5}(8 \mathrm{~mol} \%) / \mathrm{SiO}_{2}$ (92 mol \%) $(n=1.5562$ at $\lambda=1.55 \mu \mathrm{m})$ was deposited to form the core and 2nd cladding layer of the ARROW waveguide. $\mathrm{Ta}_{2} \mathrm{O}_{5}(17 \bmod \%) / \mathrm{SiO}_{2}(83 \bmod \%)(n=1.6528$ at $\lambda=1.55 \mu \mathrm{m})$ was deposited to form the core of the upper waveguide. $\mathrm{SiO}_{2}(n=1.4512$ at $\lambda=1.55 \mu \mathrm{m})$ was deposited for the 1st cladding and SLC layer of the ARROW, and the buffer separation layer between the ARROW and the upper waveguide. The waveguide pattern was formed using a reactive ion etching (RIE) with $\mathrm{CF}_{4}$. A lift-off technique was used to planarize the SLC region. The fabrication process of the SLC region is shown in Fig. 8. This process consists of the deposition of a lower half core, evaporation of a $200 \mathrm{~nm}$ Cr mask, patterning of the Cr mask with photolithography and wet chemical etching of the Cr mask with HY solution, RIE 


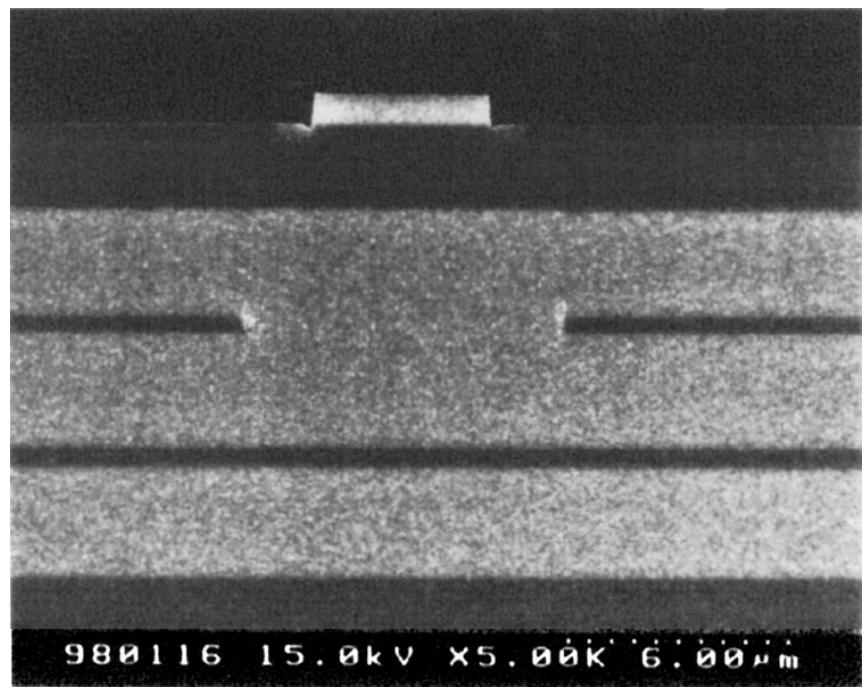

Fig. 9. SEM cross-sectional image of the fabricated device. The trench left on top of the SLC-ARROW core/cladding interface has been reduced to less than $0.1 \mu \mathrm{m}$.

etching of the lower half core to $500 \mathrm{~nm}$ depth and resputtering of a $500 \mathrm{~nm}$ thick $\mathrm{SiO}_{2}$ layer onto the $\mathrm{Cr}$ mask. In the lift-off process, the sample was first soaked into the BHF solution for $10 \mathrm{~s}$ to remove the $\mathrm{SiO}_{2}$ deposited on the vertical surface of the $\mathrm{Cr}$. Finally, the $\mathrm{SiO}_{2}$ on top of the $\mathrm{Cr}$ mask was lifted from the sample by removing the $\mathrm{Cr}$ with wet etching. Fig. 9 shows the SEM cross-sectional image of the $\mathrm{Ta}_{2} \mathrm{O}_{5} / \mathrm{SiO}_{2}$ cores of the ARROW and the upper waveguide. It can be seen in Fig. 8 that the top surface of the ARROW was kept flat with the lift-off process.

\section{EXPERIMENTAL RESULTS}

\section{A. Conventional Directional Coupler with $\theta=0^{\circ}$}

The normalized output and throughput responses of the ARROW-type VCF with $\theta=0$ and waveguide parameters which are the same as in Fig. 6 is shown in Fig. 10. It can be seen that the measured response is in good agreement with calculation. A small central wavelength shift between the two response is observed, which is probably caused by the small index distribution on the deposited film and the difference of exact shape of the upper waveguide from the rectangular shape assumed in the calculation. The full width at half-maximum (FWHM) of the measured device is $1.36 \mathrm{~nm}$ which is slightly larger than the $1.12 \mathrm{~nm}$ obtained from the calculation. This discrepancy can also be attributed to the difference between the calculated and fabricated structure. The response measured from the throughport in Fig. 10(b) shows that the extinction ratio of the filter is below $-9 \mathrm{~dB}$ as the separation thickness $t_{\text {sep }}$ of the fabricated device was approximately $0.05 \mu \mathrm{m}$ thicker than the designed value. Furthermore, scattered light could not be isolated from the detector because he lateral separation between the two waveguides at the output plane was $90 \mu \mathrm{m}$ and a single-mode fiber was used to couple light out from the waveguide to the detector. The extinction ratio of the fabricated filter might be below the measured value.

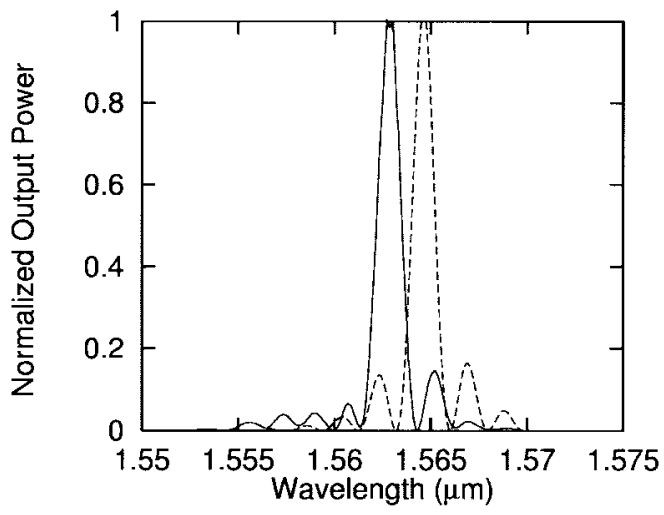

(a)

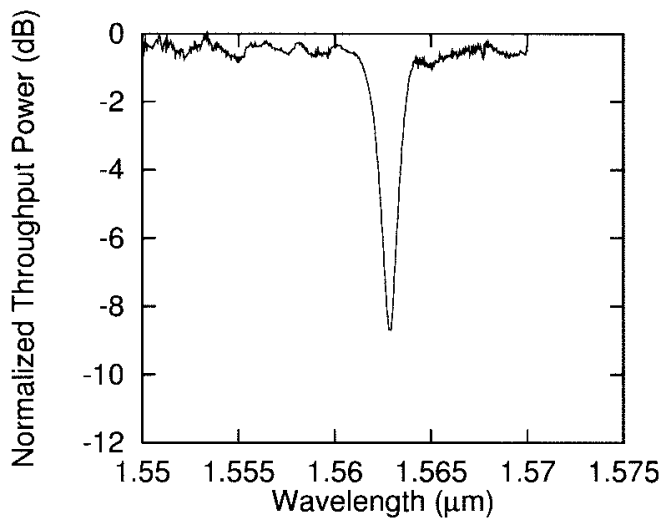

(b)

Fig. 10. Filter responses of an ARROW-type VCF with $\theta=0^{\circ}$ : (a) measured (solid line) and calculated (dashed line) of the power at the output port. (b) measured power at the throughput port. The waveguide parameters are the same as in Fig. 6.

\section{B. VCF with a Crossing Angle of $\theta$}

For a given directional coupler length, the VCF with $\theta=$ $0^{\circ}$ gives the longest coupling region, thus the narrowest bandwidth value. However, it is also difficult to detach the waveguides smoothly from each other and higher sidelobe level is resulted. The sidelobe level can be reduced at the expense of its bandwidth performance by applying apodization of the coupling strength along the device. One filter configuration that can provide a lower sidelobe level is the $\mathrm{X}$-crossing filter, where the coupling strength distribution along the length of the device turns out to resemble a hyperbolic secant function. To investigate the sidelobe level of the X-crossing ARROWtype VCF, the upper and lower waveguides of the VCF were made to cross at an angle. The crossing was fabricated in the photo lithograthy process for the upper waveguide with a slight rotation of the photo mask. The filter has a crossing angle $\theta=0.12^{\circ}$ and the buffer thickness $t_{\text {sep }}$ was reduced to $1.8 \mu \mathrm{m}$ to optimize the coupling efficiency.

Fig. 11 shows the filter responses of the $\mathrm{X}$-crossing filter. The measured FWHM and sidelobe levels are $2.88 \mathrm{~nm}$ and $-18 \mathrm{~dB}$, respectively, compared to values of $2.50 \mathrm{~nm}$ and $-35 \mathrm{~dB}$, from calculation. It can be seen that the sidelobe level was reduced with the crossing waveguides, however, the value was not nearly as low as the predicted result. One factor for the sidelobe level discrepancy could be due to 


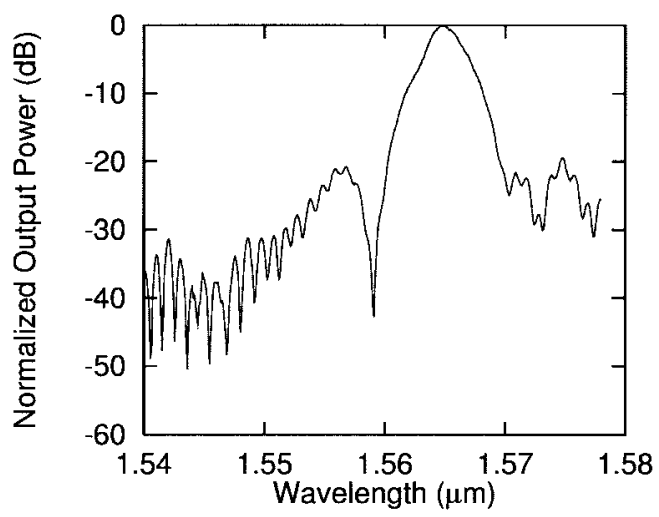

(a)

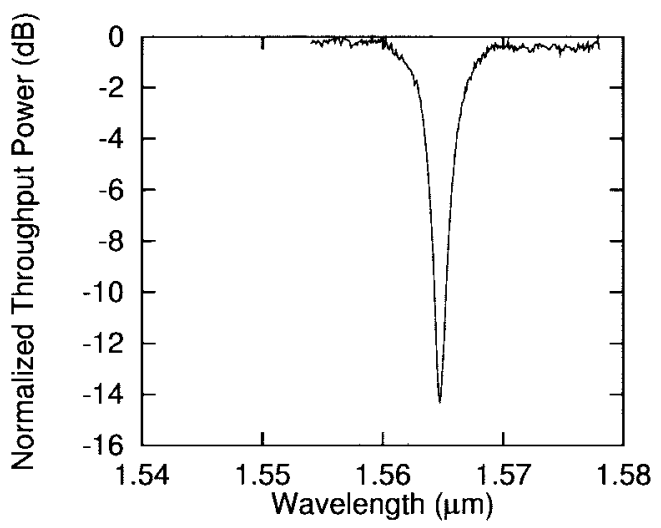

(b)

Fig. 11. Filter responses of an ARROW-type VCF with $\theta=0.12^{\circ}$, except for $t_{\mathrm{sep}}=1.8 \mu \mathrm{m}$ the waveguide parameters are the same as in Fig. 6 .

scattering from the surface roughness of the exposed ridge waveguide, although the trench depth has been reduced to less than $0.1 \mu \mathrm{m}$, nevertheless the trench shape is imprinted onto the upper waveguide when it is crossed the boundary which increases the overall scattering.

To reduce the noise from the scattered lights, a polymer cover layer with $n=1.438$ was deposited onto the device. The thickness of the upper waveguide was reduced to $t_{\text {core }}=$ $0.72 \mu \mathrm{m}$ for phase matching with the ARROW waveguide. The output response of the VCF with a cover layer is shown in Fig. 12, where the sidelobe levels are reduced to less than -23 $\mathrm{dB}$. The cover layer also reduced the differential dispersion of the waveguides and the $-3 \mathrm{~dB}$ bandwidth was increased to $3.90 \mathrm{~nm}$.

\section{CONCLUSION}

We have described a method to analysis the ARROWtype VCF and fabricated ARROW-type vertical coupler filters with $\mathrm{SiO}_{2}$ and $\mathrm{SiO}_{2} / \mathrm{Ta}_{2} \mathrm{O}_{5}$ compound glass using a lift off procedure. The filter responses of the VCF filter were found to be in very good agreement with the calculated result from the finite-difference mode solver/CMT analysis procedure. Using the conventional parallel directional coupler configuration, a narrow band filter with FWHM at $1.36 \mathrm{~nm}$ was obtained. The filter has a parallel section of $1 \mathrm{~cm}$ with the total device size of approximately $3 \mathrm{~cm}$. It is found that the

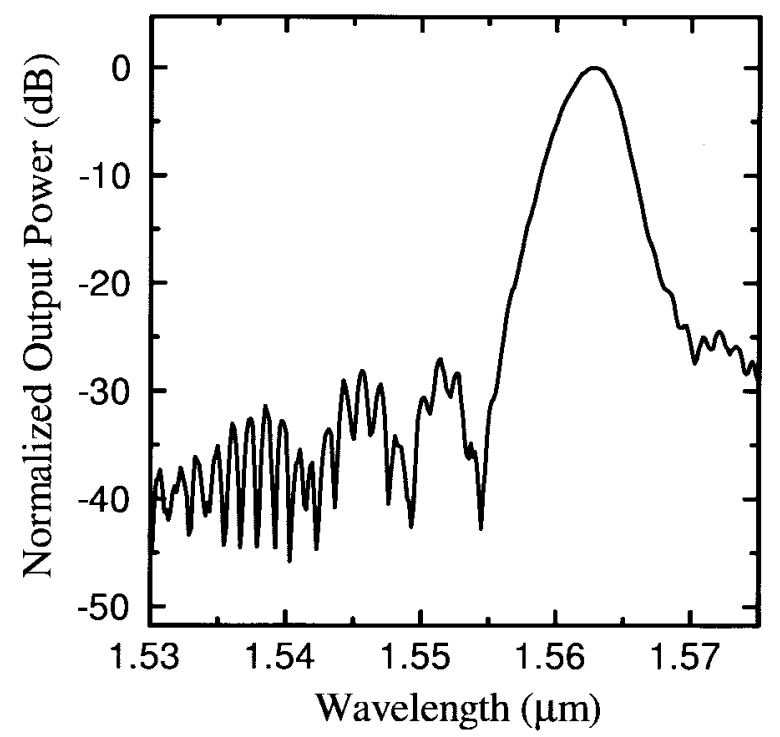

Fig. 12. Output response of the filter in Fig. 11 with a polymer cover layer with $n=1.438$, and $t_{\text {core }}=0.72 \mu \mathrm{m}$.

sidelobe level of the filter can be reduced by compromising its bandwidth. By crossing the waveguide at a small angle $0.12^{\circ}$, the sidelobe levels was suppressed to below $-18 \mathrm{~dB}$, however, the bandwidth was increased to $2.88 \mathrm{~nm}$. The sidelobe level can further be reduced by increasing the crossing angle or by reducing the noise from surface scattering with a cover layer. When applied a layer of polymer cover layer, the sidelobe levels of the VCF was reduced to below $-23 \mathrm{~dB}$. Although the FWHM of these devices is relatively large for the current DWDM systems, both the filter bandwidth and the device size can be scaled down if semiconductor materials are used.

A lift-off procedure which can reduce the surface roughness on the SLC-ARROW waveguide after its fabrication was presented. The overall performance of the ARROW-type VCF was improved from the flatness of the ARROW waveguide where coupling strength apodization can be applied to the vertical coupler filters.

\section{ACKNOWLEDGMENT}

The authors express their sincere thanks to S. Shikii of Oki Electric Company, Ltd., Japan, for supplying a fiber amplifier and S. Ozawa of Furukawa Electric Company, Ltd., Japan, for supplying optical fibers. S. T. Chu would like to thank C. L. $\mathrm{Xu}$ of Apollo Photonics Inc. for the use of the finite-difference waveguide mode solver.

\section{REFERENCES}

[1] R. C. Alferness, L. L. Buhl, U. Koren, B. I. Millar, M. G. Young, T. L. Koch, C. A. Burrus, and G. Raybon, "Broadly tunable InGaAsP/InP buried rib waveguide vertical coupler filter," Appl. Phys. Lett., vol. 60, pp. 980-982, 1992.

[2] S. T. Chu, M. Miura, and Y. Kokubun, "Compact ARROW-type vertical coupler filter," IEEE Photon. Technol. Lett., vol. 8, pp. 1492-1494, 1996.

[3] C. Wu, C. Roland, F. Shepherd, C. Larocque, N. Puetz, and J. M. Xu, "InGaAsP/InP vertical directional coupler filter with optimally designed wavelength tunability," IEEE Photon. Technol. Lett., vol. 4, pp. 457-459, 1993.

[4] C. Y. Park, D. B. Kim, T. H. Yoon, J. S. Kim, K. R. Oh, S. W. Lee, S. M. Lee, J. H. Ahn, H. M. Kim, and K. E. Pyun, "Fabrication of 
wavelength-tunable InGaAsP/InP grating-assited codirectional coupler filter with very narrow bandwidth," Electron. Lett., vol. 33, pp. 773-774, 1997.

[5] D. A. Smith, and J. J. Johnson, "Sidelobe suppression in an acoustooptic filter with a raised-cosine interaction strength," Appl. Phys. Lett., vol. 61, pp. 1025-1027, 1992.

[6] I. R. Croston, A. D. Carr, N. J. Parsons, S. N. Radcliffe, and L. J. St Ville, "Lithium niobate electro-optic tunable filter with high sidelobe suppression," Electron. Lett., vol. 29, pp. 157-159, 1993.

[7] B. E. Little, C. Wu, and W. P. Huang, "Synthesis of codirectional couplers with ultralow sidelobes and minimum bandwidth," Opt. Lett., vol. 20, pp. 1259-1261, 1995.

[8] S. T. Chu, W. Pan, S. Sato, T. Maeda, B. Little, and Y. Kokubun, "Reduction of filter sidelobe level by an X-crossing vertical coupled ARROW filter," IEEE Photon. Technol. Lett., vol. 10, pp. 391-393, 1998.

[9] B. E. Little, "Filter synthesis for coupled waveguides," J. Lightwave Technol., vol. 15, pp. 1149-1155, 1997.

[10] T. Baba and Y. Kokubun, "Dispersion and radiation loss characteristics of antiresonant reflecting optical waveguides- numerical results and analytical expressions-" IEEE Quantum Electron., vol. 28, pp. 1689-1700, 1992.

[11] C. L. Xu, W. P. Huang, M. S. Stern, and S. K. Chaudhuri, "Full vectorial mode calculation by finite difference method," Inst. Elect. Eng. Proc.-Optoelectron., vol. 141, pp. 281-286, 1994.

[12] S. Sato, W. Pan, S. T. Chu, S. Endo, S. Suzuki, and Y. kokubun, "59 nm trimming of center wavelength of ARROW-type vertical coupler filter by UV irradiation," accepted to be published in IEEE Photon. Technol. Lett., Feb./Mar. 1999.

S. T. Chu (M'90), photograph and biography not available at the time of publication.
W. Pan, photograph and biography not available at the time of publication.

S. Sato, photograph and biography not available at the time of publication.

Brent E. Little (M'95), photograph and biography not available at the time of publication.

T. Kaneko, photograph and biography not available at the time of publication.

Y. Kokubun (M'85), photograph and biography not available at the time of publication. 